\title{
Utilización de la metodología de aula invertida en una asignatura de Fundamentos de Informática
}

\author{
Begoña del Pino, Beatriz Prieto, Alberto Prieto, Francisco Illeras \\ Departamento de Arquitectura y Tecnología de Computadores \\ Universidad de Granada \\ \{bpino, beap, aprieto, filleras\}@ugr.es
}

\begin{abstract}
Resumen. En este trabajo presentamos la experiencia llevada a cabo durante el primer año de desarrollo de un proyecto de innovación docente financiado por la Universidad de Granada, en el que se ha aplicado la metodología de aula invertida utilizando los recursos propios de un MOOC (Massive Open Online Course), tales como videoclases, cuestionarios de auto-evaluación y foros de debate. Tras describir brevemente la plataforma tecnológica y la metodología docente, mostramos los principales resultados obtenidos de la encuesta de opinión realizada por los estudiantes y la mejora alcanzada en las calificaciones académicas con respecto a cursos anteriores. Finalmente, exponemos algunas conclusiones con la finalidad de seguir mejorando, no sólo los resultados académicos sino también el grado de satisfacción de estudiantes y profesores al utilizar este método.
\end{abstract}

Palabras Clave: Aula invertida, Clase invertida, Aprendizaje invertido, Lecciones aprendidas, Docencia aprendida, Enseñanza semi-presencial, MOOC, MOODLE, Fundamentos de Informática.

\begin{abstract}
In this paper, we present the experience carried out during the first year of a teaching innovation project supported by the University of Granada. The aim of the project is to apply the Flipped Classroom methodology taking advantage of the learning resources included in a MOOC (Massive Open Online Course), such as video-lectures, self-assessment tests and discussion forums,. After a brief description of both the technological platform and the learning methodology, we show the main results obtained from a survey of students and the academic outcomes. Finally, we draw several conclusions in order to continue improving not only the academic results, but also the satisfaction degree of students and teachers with the application of the method.
\end{abstract}

Keywords: Flipped Classroom, Flipped Learning, Blended Learning, Lessons Learned, MOOC, MOODLE, Fundamentals of Computer Systems. 


\section{Introducción}

El método de aula invertida (Flipped Classroom) es un sistema de aprendizaje en que el estudiante debe haber estudiado la materia correspondiente con antelación a la clase presencial mediante vídeos en los que se exponen los diferentes conceptos. Después asiste a la clase para aclarar dudas, relacionar y reforzar conceptos, y realizar ejercicios prácticos [1,2,3]. Se invierte, por tanto, la dinámica respecto a la metodología más tradicional en la que el profesor expone la materia en clase, y con posterioridad el estudiante estudia el contenido y realiza ejercicios y tareas en casa como complemento a su estudio.

La metodología del aula invertida es una de las técnicas de enseñanza y aprendizaje con mayor proyección de futuro $[4,5]$. Se relaciona con los conceptos de docencia aprendida (Lessons Learned) y enseñanza semi-presencial (Blending learning [6]). El estudiante debe haber estudiado previamente la materia, y lo hace por medio de materiales disponibles en línea.

El éxito de esta metodología depende obviamente de la calidad del material disponible en internet, y también de la apropiada organización de las clases presenciales en las que el profesor debe detectar las dificultades en el aprendizaje previo de los estudiantes, y utilizar los recursos idóneos para corregir los errores de comprensión que se hayan producido y, en definitiva, guiar el aprendizaje de un grupo de estudiantes que puede ser muy numeroso y heterogéneo, fomentando la interacción estudiante-profesor y procurando una atención personalizada [7]. No obstante, hay otros factores que también influyen en el rendimiento de los estudiantes durante el curso, como por ejemplo su grado de aceptación hacia una metodología que exige una dedicación constante para poder participar y seguir con aprovechamiento las clases.

Durante este curso académico 2015-2016 se ha aplicado esta metodología en la asignatura "Fundamentos de Informática" (Tabla 1) del primer curso de las Titulaciones de Grado de Ingeniería en Tecnologías de Telecomunicación (105 estudiantes matriculados) y Grado de Ingeniería Electrónica Industrial (74 estudiantes) de la Universidad de Granada. Los estudiantes de estas asignaturas han utilizado en línea el material disponible en un MOOC de la plataforma abiertaUGR [8], en el que además han participado de forma no presencial otros 116 estudiantes de diferente procedencia.

\section{Antecedentes}

Con objeto de mejorar los resultados académicos, en el curso pasado 2014-2015 se realizaron vídeos a partir de las presentaciones que se venían utilizando en clases presenciales expositivas más tradicionales, con la idea de que este medio resultase más atractivo a los estudiantes, acostumbrados a desenvolverse cotidianamente en un mundo digital. Se les propuso visionar las lecciones con antelación a las clases presenciales, con el propósito de disponer también de más tiempo para realizar problemas en el aula y resolver dudas. Además, fue posible realizar 5 tests de evaluación al finalizar los diferentes temas teóricos durante las propias clases 
presenciales (en cursos anteriores se realizaba un único test de teoría al final del cuatrimestre).

En enero de 2015 se habían publicado 37 videoclases en Youtube, que en el momento de redactar este artículo han superado las 70.000 visualizaciones con una duración total de 477.692 minutos. Si bien el $57 \%$ de las visitas proceden de España, el $43 \%$ restante procede de otros 84 países.

Con esta experiencia mejoraron las calificaciones obtenidas por los estudiantes con respecto a cursos anteriores, y además, ante el interés suscitado por los vídeos del curso en la web, surgió la idea de diseñar un MOOC complementando las videoclases con otros materiales docentes para el aprendizaje, la auto-evaluación y la tutorización de los participantes.

Tabla 1. Descripción resumida de la asignatura "Fundamentos de Informática" de acuerdo con la guía docente.

\section{Breve descripción de contenidos (según memoria de verificación de grado):}

Estructura funcional de los ordenadores. Concepto y uso de Sistema Operativo. Concepto y uso de Base de Datos. Elementos de programación. Herramientas informáticas con aplicación en Ingeniería.Trabajo aislado de los alumnos.

\section{Objetivos (expresados como resultados esperables de la enseñanza):}

- Entender el significado global de la Informática.

- Comprender cómo se representa la información en el interior de un computador.

- Conocer la estructura funcional de un computador.

- Comprender el concepto de programación y enumerar sus principales características.

- Comprender el funcionamiento de un computador a nivel de lenguaje máquina y lenguaje ensamblador.

- Conocer los fundamentos de los traductores: compiladores e intérpretes.

- Analizar la funcionalidad de un sistema operativo en cuanto a la gestión de procesos, gestión de memoria, gestión de entradas/salidas y gestión de archivos.

- Manejar adecuadamente los sistemas operativos más comunes en la actualidad.

- Entender el concepto de base de datos.

- Diseñar bases de datos relacionales sencillas e implementarlas en un sistema gestor de bases de datos.

- Conocer y aplicar herramientas informáticas específicas de las ingenierías, como aplicaciones para cálculo matemático, representación científica de información y simulación de sistemas.

\section{Plataforma tecnológica}

Los recursos docentes que utilizan los estudiantes de las asignaturas de "Fundamentos de Informática" se encuentran en un MOOC (descrito con detalle en 
[9]) en la plataforma de formación abierta online de nuestra universidad. Se proporcionan diversos materiales docentes estructurados por temas de teoría, encuestas de opinión y estrategias de gamificación basadas en la obtención de insignias para incentivar la participación y seguimiento durante el cuatrimestre.

Para cada tema (Figura 1) se dispone de las videoclases junto con las correspondientes presentaciones en texto, relaciones de problemas propuestos y resueltos, algunas tareas complementarias destinadas a la búsqueda de información actual en relación con cuestiones de particular interés en la asignatura, tests de autoevaluación y un foro de debate, en el los estudiantes plantean y resuelven sus propias dudas bajo la supervisión de los profesores.

Asimismo, se han realizado tres encuestas sobre la situación y conocimientos previos del estudiante, el grado de satisfacción con la metodología docente seguida en la asignatura, y sobre la labor docente del profesorado.

Se han concedido insignias parciales ligadas fundamentalmente a la realización de actividades de cada tema (exigiendo que se hayan superado los tests de autoevaluación con una calificación mínima de 8 puntos sobre 10) y una insignia final el seguimiento del curso completo, que nos proponemos registrar en el sistema Mozilla Open Badges. ${ }^{1}$

\section{Metodología docente}

La metodología seguida en la asignatura se basa en las siguientes acciones formativas:

- Videoclases expositivas de la materia

- Clases presenciales de debate y resolución de problemas

- Clases prácticas y seminarios

- Tutorías

Los contenidos teóricos correspondientes a cada tema se transmiten a los estudiantes de forma sistemática mediante la visualización de las lecciones en vídeo, fomentando de este modo, su capacidad para estudiar y aprender nuevos conceptos de forma autónoma. Después, en las clases presenciales el profesor plantea ciertas cuestiones a través de las cuales descubre las posibles dudas y dificultades, refuerza e interrelaciona los conceptos principales y propone la realización de problemas aplicados a casos reales. Se busca interpelar al estudiante, proponiéndole nuevos puntos de vista para que se forme en una mentalidad crítica.

En las clases prácticas y seminarios los estudiantes aplican los conocimientos teóricos mediante la instalación y uso de una distribución GNU-Linux, el diseño de bases de datos relacionales sencillas con un sistema gestor de bases de datos, el uso de herramientas informáticas con aplicación en la ingeniería (MATLAB), y la realización de ejercicios básicos de programación con lenguajes de alto nivel (MATLAB o C) y de programación en código máquina y ensamblador para un procesador didáctico elemental. Estas actividades se realizan de forma presencial en grupos reducidos (de 20-25 estudiantes, aproximadamente).

\footnotetext{
${ }^{1}$ https://support.mozilla.org/es/products/open-badges
} 


\section{Tema 1: Conceptos elementales de Informática.}

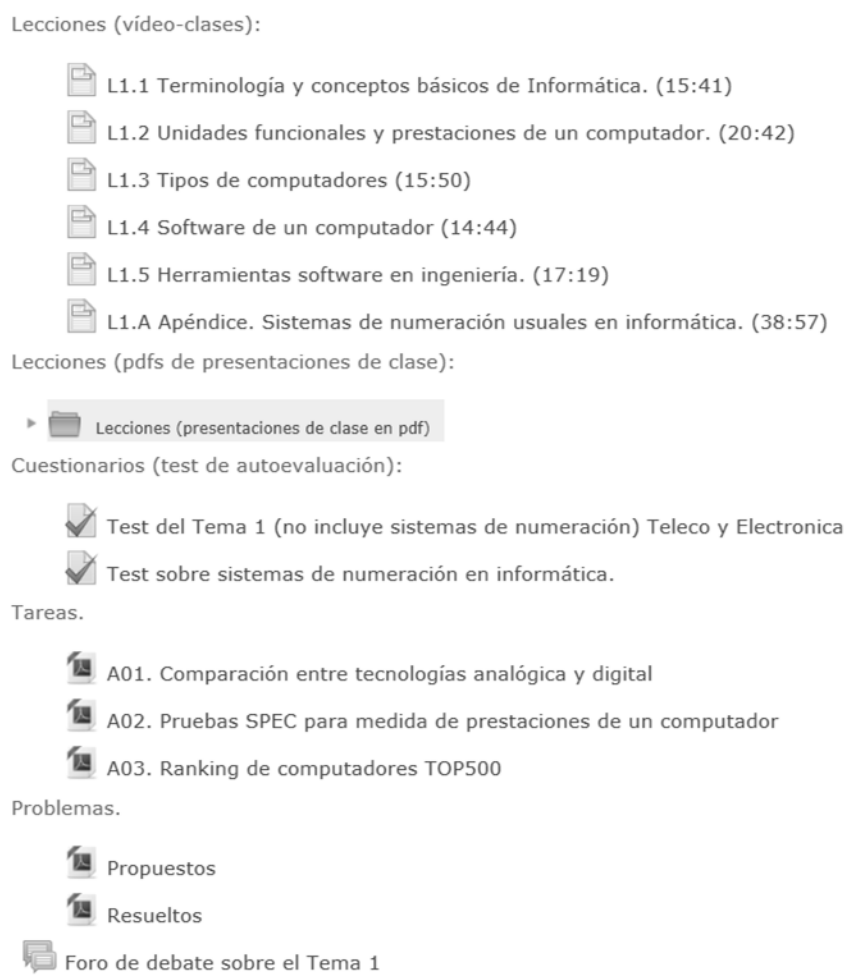

Figura 1. Ejemplo de materiales docentes proporcionados en el MOOC en relación con el Tema 1.

La tutorízación se realiza de forma presencial en el despacho de los profesores en un horario establecido para atención a los estudiantes y también de forma no presencial, no sólo respondiendo dudas planteadas directamente al profesor, sino también supervisando los debates que se suscitan en los foros.

En este curso académico, el método de aula invertida sólo se ha empleado para las actividades relacionadas con los contenidos teóricos, no para las prácticas y seminarios. Cada semana el profesor indica por internet a los estudiantes de su grupo las videoclases que debe estudiar con antelación, junto con las referencias bibliográficas que puede consultar (fundamentalmente [10] y [11]) y algunos problemas propuestos en la plataforma que puedan estar relacionados. Las actividades se planifican para que el tiempo semanal de trabajo autónomo del estudiante no supere las 4 horas, de acuerdo con los créditos asignados a la asignatura en la guía docente. 
Al finalizar los temas de teoría, se propone a los estudiantes que realicen los cuestionarios de test disponibles en la plataforma web para su auto-evaluación (de entre un total de 305 preguntas, se generan cuestionarios de 10 preguntas de respuesta múltiple ordenadas aleatoriamente), y se determina con antelación suficiente la fecha en que se realiza un cuestionario de test durante la clase presencial. Las preguntas en los tests presenciales tienen una dificultad similar a la de los tests de autoevaluación.

\section{Resultados}

A continuación resumimos los principales resultados en cuanto a la valoración de la metodología docente por parte de los estudiantes (según la encuesta de opinión disponible en la plataforma) y en cuanto a las calificaciones académicas obtenidas en la convocatoria ordinaria de este curso con respecto a cursos anteriores.

\subsection{Seguimiento por parte de los estudiantes}

Aunque desde el principio del cuatrimestre se hizo hincapié en que se debían dedicar al trabajo autónomo 4 horas semanales, tan sólo el 15\% afirma haber dedicado en media entre 3 y 4 horas semanales al estudio de la asignatura. El 69\% le ha dedicado entre 1 y 3 horas por semana, y un $6 \%$ más de 4 horas.

Por otro lado, un 15\% manifiesta haber preparado entre el $60 \%$ y el $80 \%$ de las actividades propuestas semanalmente, y sólo un $12 \%$ afirma haber preparado más del $80 \%$ de las actividades.

\subsection{Valoración de la metodología del aula invertida}

El $63 \%$ considera que esta metodología le ha sido "bastante" o "muy" útil para comprender los conceptos, y el 59\% opina que le ha sido "bastante" o "muy" útil para aprender a solucionar los ejercicios propuestos. El 74\% afirma que la metodología fomenta el auto-aprendizaje "mucho" o "bastante".

Un porcentaje del $59 \%$ prefiere en general la metodología del aula invertida frente a las clases presenciales expositivas tradicionales. Al preguntar sobre las principales ventajas e inconvenientes del método del aula invertida se observa que los estudiantes aprecian la ventaja de poder acceder a los vídeos por internet en cualquier momento, y tantas veces como sea necesario repitiendo el visionado de las partes de mayor dificultad. Sin embargo, aparecen opiniones muy diversas en cuanto al papel de las clases presenciales. La mayor parte de las respuestas valoran positivamente que las clases presenciales sean más dinámicas y que se puedan realizar más ejercicios; otros en cambio cuestionan el sentido de asistir a clase: unos por considerar que ya se ha estudiado previamente la materia; y otros porque no pueden aprovechar el tiempo si por el contrario no se han preparado las tareas con antelación. 


\subsection{Valoración de los materiales y tecnologías disponibles en la plataforma online}

Los recursos más utilizados y mejor valorados han sido los tests de autoevaluación (un 97\% los ha utilizado "bastante" o "mucho", y el 91\% los considera "bastante" o "muy" útiles), seguidos de las videoclases (utilizadas "mucho" o "bastante" por el $80 \%$, y valoradas como "muy" o "bastante" útiles por el 69\%). Cabe destacar que sólo el $52 \%$ dice haber utilizado "mucho" o "bastante" los foros de debate, mientras que el $65 \%$ los considera "bastante" o "muy" útiles para su aprendizaje.

\subsection{Valoración de las clases presenciales}

Las clases presenciales son consideradas "bastante" o "muy" útiles para comprender y afianzar los conceptos presentados en el material docente por el 65\%, y para aprender a realizar ejercicios por el $71 \%$.

\subsection{Valoración del curso en general}

El porcentaje de estudiantes que indican un grado de satisfacción "bastante" o "mucho" con la metodología del aula invertida es del 66\%; con el material docente del 83\%; con las clases presenciales del 66\%; y con el curso en general del $79 \%$.

\subsection{Mejora en los resultados académicos}

En los últimos tres cursos académicos el porcentaje de estudiantes que aprueba la asignatura (con respecto al total de matriculados) ha pasado del 57,25\% al 82,70\%, y la nota media final ha pasado de 5,32 $\pm 1,56$ a $6,74 \pm 1,59$. La nota final se ha obtenido como la suma ponderada de las notas obtenidas en los cuestionarios de test presenciales $(30 \%)$, en el examen de problemas realizado al final del cuatrimestre $(50 \%)$ y de las actividades propuestas en prácticas y seminarios $(20 \%)$.

\section{Conclusiones}

Durante el presente curso académico 2015-2016 se ha aplicado la metodología de aula invertida en la asignatura de "Fundamentos de Informática" de las Titulaciones de Grado en Ingeniería Electrónica Industrial e Ingeniería en Tecnologías de Telecomunicación utilizando los recursos propios de un MOOC disponible en la plataforma de formación abierta online de la Universidad de Granada. Esta experiencia se ha realizado en el marco de un proyecto de innovación docente financiado por nuestra Universidad de dos años de duración. Presentamos a continuación las principales conclusiones a las que hemos llegado a la vista de los resultados obtenidos, con el fin de tratar de mejorar la experiencia de cara al próximo curso: 
No se ha conseguido en general que, de forma constante durante el cuatrimestre, los estudiantes dediquen un tiempo de estudio semanal suficiente para realizar las tareas asignadas. Aparte de la capacidad de motivación en las clases presenciales, otros factores externos pueden contribuir a explicar esta situación.

En cada curso de las dos titulaciones involucradas en la experiencia, esta es la única asignatura que emplea el método del aula invertida. Por otro lado, los estudiantes son de primer curso y primer cuatrimestre por lo que aún están en fase de adaptación a sus nuevos estudios de educación superior. Además, se ha observado la interferencia de otras asignaturas en un descenso en la asistencia a clase o falta de preparación de la misma, coincidiendo con semanas en las que los estudiantes estaban convocados para realizar otras pruebas o exámenes en el marco de la evaluación continua.

El método resulta muy exigente tanto para el estudiante como para el profesor, que en ocasiones se encuentra en la disyuntiva de "presentar los conceptos principales" para contextualizar a quienes no han podido realizar las tareas semanales (a costa de aburrir a quienes han realizado un cumplimiento adecuado) o "respetar" lo establecido asumiendo que una parte de los estudiantes no van a poder participar adecuadamente en las actividades propuestas para la clase presencial. Una coordinación más estrecha entre asignaturas de un mismo curso podría ayudar a planificar las actividades semanales (para antes y durante la clase presencial) de una forma más flexible.

Aunque parece que las clases presenciales han cumplido en general con el objetivo de reforzar conceptos y ayudar a resolver ejercicios, será conveniente explorar nuevas técnicas que fomenten la participación interactiva de los estudiantes para aumentar su motivación y tratar así de que se sientan más comprometidos con el seguimiento continuado de la metodología.

En general, los recursos docentes que se han utilizado han sido bien valorados, lo que nos reafirma en que el uso de los recursos del MOOC ha sido un acierto, si bien en la pregunta de respuesta libre sobre inconvenientes de las videoclases, la mayoría coincide en señalar su excesiva duración, por lo que será necesario acortarlas para próximas ediciones.

A la vista de la notable mejora que se ha producido en las calificaciones obtenidas por los estudiantes al realizar pruebas de evaluación semejantes en estructura, contenido y dificultad a las de cursos académicos anteriores, podemos afirmar que utilizar el método de aula invertida junto con los recursos de un MOOC permite conjugar de forma muy positiva la enseñanza a distancia y la enseñanza presencial.

Agradecimientos. La experiencia descrita se desarrolla en el marco del Proyecto de Innovación Docente 15-82 financiado por la Unidad de Calidad, Innovación y Prospectiva de la Universidad de Granada. Nuestro agradecimiento también al Centro de Enseñanzas Virtuales de esta universidad (CEVUG) por su apoyo y asesoramiento.

\section{Referencias}

1. Alvarez, A.; Flipping the Classroom: Homework in Class, Lessons at Home. Education Digest: Essential Readings Condensed for Quick Review 77(8) 18-21, (2012). 
1. Bergmann, J.; Overmyer, J.; Wilie, B.: The flipped class: Myths vs. reality. The Daily Riff, 1-4. (2011)

2. Tucker, T.: The flipped classroom. Education Next 12 (1), 2012.

3. Pearson. Ideas que inspiran. Cinco técnicas de enseñanza que están revolucionando las aulas. http://ideasqueinspiran.com/2015/10/08/cinco-tecnicas-de-ensenanza-que-estanrevolucionando-las-aulas/ 8 octubre (2015).

4. Rosenberg, T.: Turning Education Upside Down. New York Times.9 Octubre (2013).

5. Garrison, D.R.; Heather Kanuka. Blended learning: Uncovering its transformative potential in higher education. The internet and higher education 7.2: 95-105, (2004).

6. Kim, M.K..; Kim, S.M.; Khera, O.; Getman, J.: T The experience of three flipped classrooms in an urban university: an exploration of design principles. The Internet and Higher Education, Vol. 22, pp. 37-50, July (2014).

7. Curso MOOC "Fundamentos de Informática" en la plataforma abiertaUGR: https://abierta.ugr.es/course/view.php?id=10. (2016)

8. Prieto, A.; Prieto, B.; del Pino, B.; Illeras, F.: Sinergias entre MOOC y Flipped Classroom: una experiencia. Experiencia MOOC: Un enfoque hacia el aprendizaje digital, la creación de contenidos docentes y comunidades online; M. Gea (Edt.); Editorial Universidad de Granada; pp.133-154, (2016).

9. Prieto, A.; Beatriz Prieto, B.: Conceptos de Informática. Serie Schaum, McGraw-Hill (2005).

10. Prieto, A.; Lloris, A.; Torres, J.C.: Introducción a la Informática, 4a Edición. McGrawHill. (2006). 